\title{
Computer Research of Building Materials
}

\author{
Olga Vasil'eva* \\ Moscow State University of Civil Engineering, Yaroslavskoye shosse, 26, Moscow, 129337, Russia
}

\begin{abstract}
A computer study of the technological processes for the production of building materials based on the use of reversible autocatalysis reactions is carried out. For the mathematical description of the process of autocatalytic reactions, mathematical models are used, described by a initial-boundary problems for the Carleman system. The considered mathematical model describes the autocatalysis reaction under the following assumptions: the rate of decrease in the density of positive ions is proportional to the square of the deviation of the density of positive ions from the equilibrium state, the rate of decrease in the density of negative ions is proportional to the square of the deviation of the density of negative ions from the equilibrium position and the law of conservation of the total charge of the system including liquid solution. This mathematical model is non-linear, therefore numerical methods are used for its study. For a numerical solution of the initial-boundary value problem for the Carleman system, a finite-difference method of the second order of accuracy is used. For the numerical investigation of the solution obtained, linear interpolation and spline interpolation are used. A numerical study is made on the dependence of the chemical reaction time from the parameters of the model and various initial conditions.
\end{abstract}

\section{Introduction}

A computer study of the technological processes for the production of building materials based on the use of autocatalysis reactions is carried out. For the mathematical description of the process of autocatalytic reactions, mathematical models are used, described by a initial-boundary problem for the Carleman kinetic system of equations [1-8]. For simplicity of exposition and the possibility of comparing and verifying the obtained numerical results, we will consider mathematical models formulated for the reduced variables.

\section{Formulation of the problem}

We consider the mathematical model describes the following reversible autocatalysis reaction. A narrow vessel of length $1(0 \leq x \leq 1)$ is filled with a liquid solution that is a source of positive and negative ions $\left(\mathrm{R}^{+}, \mathrm{S}^{-}\right)$in the reaction. On the left boundary of the vessel $(x=0)$, the time dependence of the density of positive ions is given, at the right boundary of the vessel $(x=1)$, the time dependence of the density of negative ions is

\footnotetext{
* Corresponding author: vasilievaOA@mgsu.ru
} 
specified. Positive and negative ions move with constant equal modulo $c>0$ and oppositely directed velocities. In the court there is a reversible chemical reaction

$$
\mathrm{P}+\mathrm{R}^{+}=\mathrm{S}^{-}, \mathrm{P}+\mathrm{S}^{-}=\mathrm{R}^{+}
$$

where $\mathrm{P}$ is the liquid solution, $\mathrm{R}^{+}, \mathrm{S}^{-}$is the positive and negative ions.

We denote by $u_{d}(t, x)$ and $w_{d}(t, x)$ the densities of ions of types $\mathrm{R}^{+}, \mathrm{S}^{-}$at a point with coordinate $x$, at time $t$, and $u_{s}, w_{s}$ are the stationary concentrations at which the reaction terminates. We denote the deviations of the densities from the stationary densities by $u(t, x)$ $=u_{d}(t, x)-u_{s}$ and $w(t, x)=w_{d}(t, x)-w_{s}$.

The mathematical model of the process is the initial-boundary problem for the Carleman system of equations

$$
\begin{aligned}
& \frac{\partial u}{\partial t}+c \frac{\partial u}{\partial x}=-\frac{1}{\varepsilon}\left(u^{2}-w^{2}\right), \quad 0<x<1, \quad t>0 \\
& \frac{\partial w}{\partial t}-c \frac{\partial w}{\partial x}=\frac{1}{\varepsilon}\left(u^{2}-w^{2}\right)
\end{aligned}
$$

with initial conditions

$$
\begin{aligned}
& \left.u\right|_{t=0}=u^{0}(x), \\
& \left.w\right|_{t=0}=w^{0}(x)
\end{aligned}
$$

and the agreed boundary conditions

$$
\begin{gathered}
u(t, 0)=u_{0}(t), w(t, 1)=w_{1}(t) \\
u_{0}(0)=u^{0}(0), w_{1}(0)=w^{0}(1)
\end{gathered}
$$

It is assumed that the initial conditions satisfy the relations:

$$
\int_{0}^{1} u^{0}(x) d x=0, \int_{0}^{1} w^{0}(x) d x=0 .
$$

The mathematical model describes the autocatalysis reaction under the following assumptions: the rate of decrease of the density $\mathrm{R}^{+}$is proportional to $u^{2}(t, x)=\left(u_{d}(t, x)-u_{s}\right)^{2}$, the rate of density decrease $\mathrm{S}^{-}$is proportional to $w^{2}(t, x)=\left(w_{d}(t, x)-w_{s}\right)^{2}$ and the law of conservation of the total charge of the system including liquid solution is satisfied.

\section{Numerical results}

We consider the mathematical model (1) - (3) for the following initial-boundary conditions $u^{0}(x)=A \sin (2 \pi \omega x), w^{0}(x)=0, u_{0}(t)=0, w_{0}(t)=0$ and for different values of the parameters $\varepsilon, A, \omega, c$. For the numerical investigation of the problem we use the finitedifference scheme of second order of accuracy linear and spline approximations. The solution of the problem tends to a zero stationary solution for $t \rightarrow \infty$ (stabilizes) for sufficiently small deviations from zero of the initial conditions [7]. 
To estimate the stabilization time of the solution (reaction time), we introduce the following notation. Denote by $D u(t)$ the maximum deviation on the segment $[0,1] u(t, x)$ from the zero equilibrium position and $D w(t)$ the maximum deviation on the segment $[0,1]$ $w(t, x)$ from the zero equilibrium position. So,

$$
D u(t)=\max _{0 \leq x \leq 1}|u(t, x)|, \quad D w(t)=\max _{0 \leq x \leq 1}|w(t, x)| .
$$

we define the stabilization time (the reaction time) $T$ for the level $\alpha$ to be the value of the time variable $t$ such that for any $t>T$

$$
\max \{D u(t), D w(t)\} \leq \alpha \max \{D u(0), D w(0)\} .
$$

Figure 1 shows the time dependence of the maximum deviations for different values of frequency $\omega, A=0.02, c=1$ and $\varepsilon=0.02$. The curves du1, du2, du3, du5, du 7, du10, du20 correspond to the following values $\omega: 1,2,3,5,7,10$, 20. In Fig. 1, we can see that for small frequencies, the time dependence of maximum deviations is not monotonous. For $\omega=$ 2 , a phenomenon similar to resonance is observed, with decreasing $\varepsilon$, leading to instability.

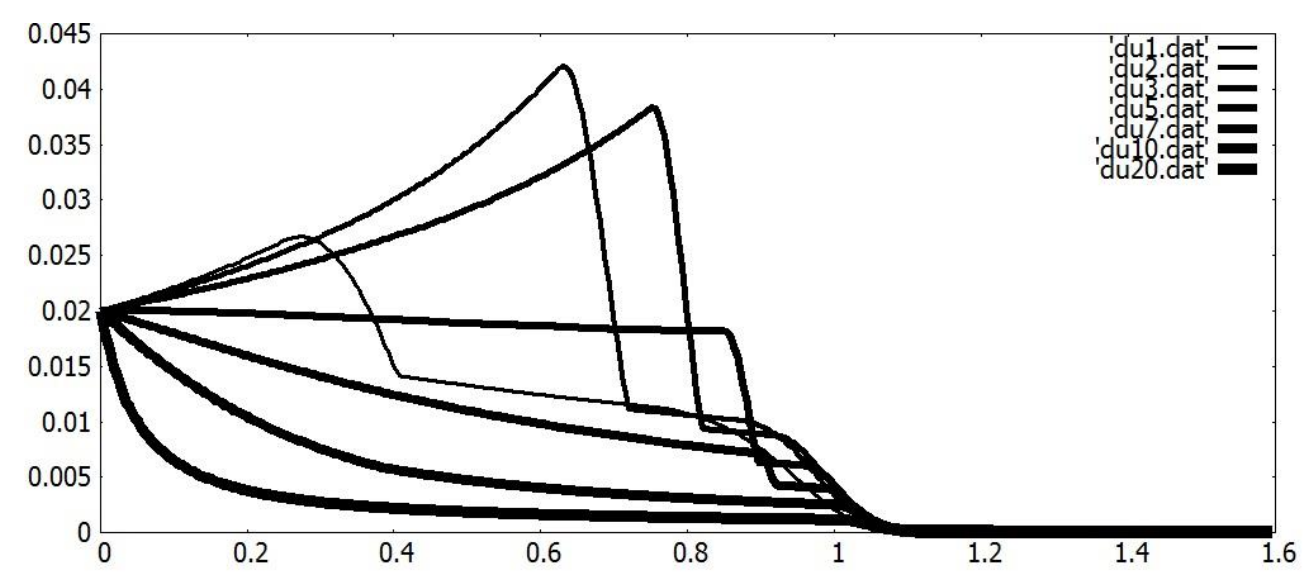

Fig. 1. The time dependence of the maximum deviations for different values of frequency.

Reducing the particle propagation velocity modulo to $\mathrm{c}=0.75$ does not lead to a change in the nature of the dependence of the maximum deviation on time. However, the stabilization time at low frequencies increases noticeably. Fig. 2 shows the time dependences of the maximum deviation from the equilibrium position for different frequencies and different particle velocities. The curves du1, du5, du10 correspond to the following values of omega: $1,5,10$ and particle velocities modulo $c=1$, the curves du11, du51, du101 correspond to the values of omega: 1, 5, 10 and particle velocities modulo $c=0.75$. 


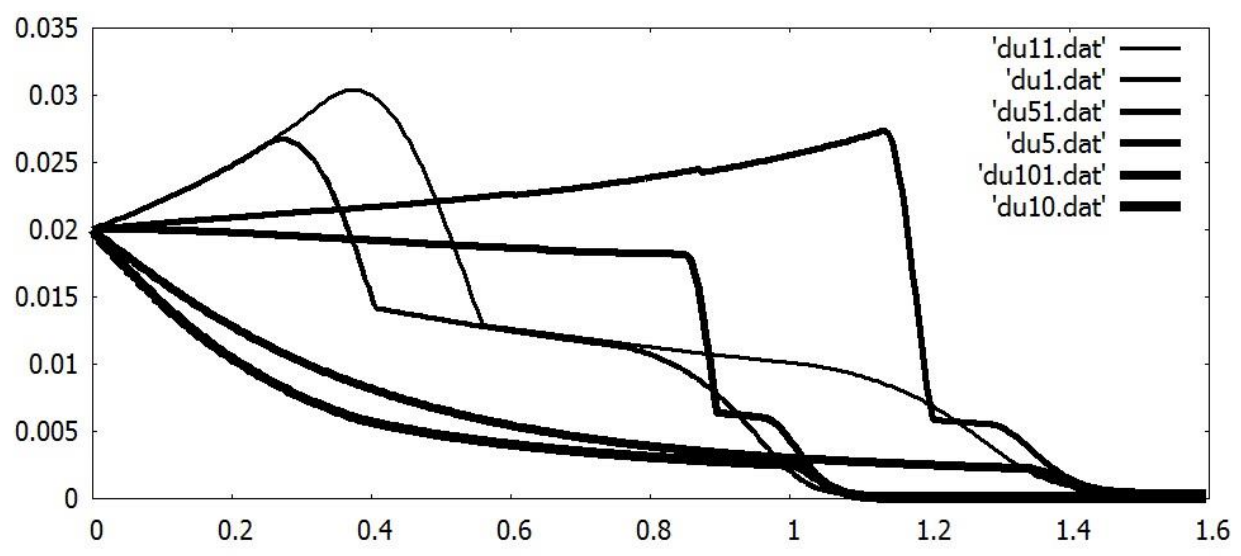

Fig. 2. The time dependence of the maximum deviations for different values of frequency and velocities.

Figure 3 shows the stabilization time dependence on $\omega$ for different values $\alpha$. The curves T1, T2, T3 correspond to the following $\alpha: 0.1,0.05,0.03$, the parameters $A=0.02$, $\varepsilon=0.02$ and $c=1$. For large frequencies $\omega>16$ we can see a rapid decrease of the stabilization time for $\alpha=0.1$. The time of stabilization of the solution for $\alpha=0.05$ and $\alpha=$ 0.03 is practically independent of frequency.

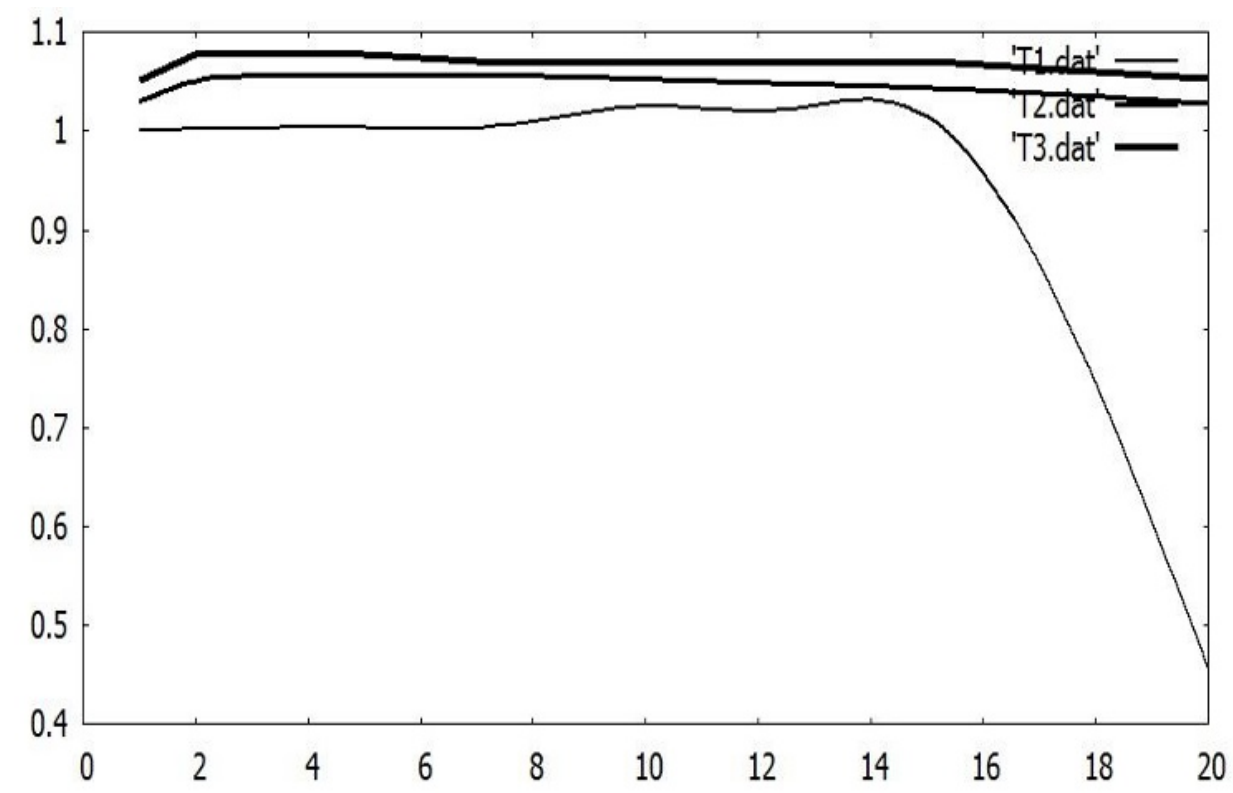

Fig. 3. The stabilization time dependence from parameter $\omega$ for different values $\alpha$.

Fig. 4 shows the stabilization time dependence from $\varepsilon$ for different values $\alpha$. The curves T1, $\mathrm{T} 2$, T3 correspond to the following $\alpha: 0.1,0.05,0.03$, the parameter $A=0.02, \omega=1, c=1$. 


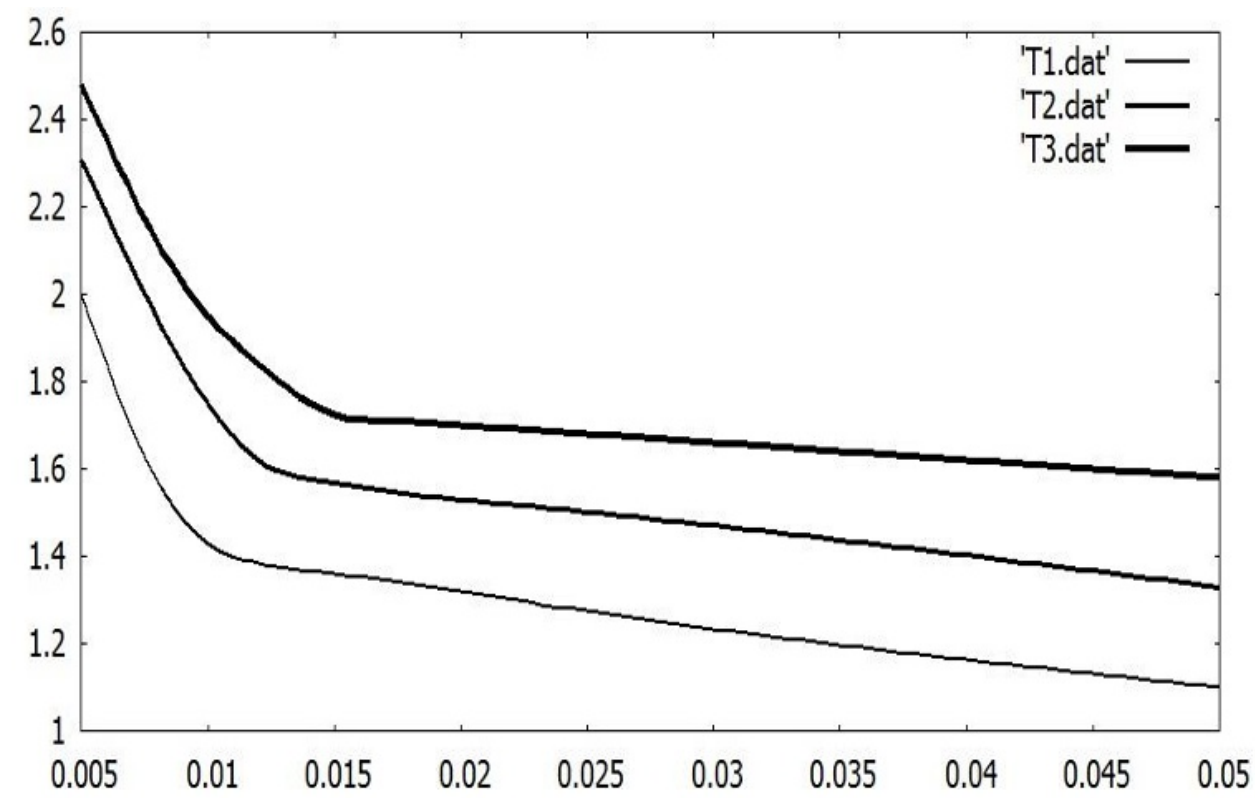

Fig. 4. The stabilization time dependence from parameter $\varepsilon$ for different values $\alpha$.

The dependence of the reaction time on the parameter $A$ (the maximum deviation of the initial conditions from the zero equilibrium position) can be obtained by making the change of variables $u_{1}(t, x)=(0.02 / A) u(t, x), \quad w_{1}(t, x)=(0.02 / A) w(t, x)$. Then the problem reduces to the original one with the new parameter $\varepsilon_{1}=\varepsilon(0.02 / A)$. Thus, the reaction time with the parameter of the maximum deviation of the initial conditions from the zero equilibrium position equal to $p A$ and the parameter $p \varepsilon$ is equal to the reaction time with the parameter of the maximum deviation of the initial conditions from the zero equilibrium position $A$ and the parameter of the problem $\varepsilon$.

\section{Conclusions}

We consider the mathematical model describes the reversible autocatalysis reaction. The mathematical model of the process is the initial-boundary problem for the system of Carleman equations. There is not analytical solution of the system. So, for it investigation we use a finite-difference method of the second order of accuracy is used. For the numerical investigation of the solution obtained, linear interpolation and spline interpolation are used. The results of numerical investigation of the dependence of the chemical reaction time on the parameters of the model and various initial conditions is presented and discussed. A detailed study of the reaction time dependence on the particle velocities, as well as, the consideration of a two-dimensional problem are the subjects of the following publications

The author would like to express gratitude to Professor E.V.Radkevich for formulation of the problem and useful discussions and comments. 


\section{References}

1. O. Vasil'eva, Matec Veb. Conf. 25104936 (2018)

2. S.K.Godunov, U.M.Sultangazin, Math. Surv 26 pp 3-51 (1971)

3. E.V.Radkevich, J. Math. Sci 181 pp 232-80 (2012)

4. E.V.Radkevich, J. Math. Sci 181 pp 701-50 (2012)

5. V.V.Aristov, O.V.Ilyin, $27^{\text {th }}$ International Symposium on Rarefied Gas Dynamics 2010, RGD27 (AIP Conference proceeding) pp 649-654 (2010)

6. E.V.Radkevich, O A.Vasil'eva, S.A. Duhnovskij J. Math. Sci 201 pp 296-32 (2015)

7. E.V.Radkevich, O.A.Vasil'eva J. Math. Sci 224 pp 1-32 (2017)

8. O.A.Vasil'eva VestnikMGSU 12 pp 23-33 (2016) 